\title{
A NECESSARY CONDITION TO THE SIMULTANEOUS CORRECTION \\ OF CELESTIAL REFERENCE FRAMES AND MINOR PLANETS \\ ORBITAL ELEMENTS
}

\author{
M.J. MARTÍNEZ USÓ \\ Departamento de Matemática Aplicada. ETSII, Universidad Politécnica de Valencia, \\ SPAIN. \\ and \\ F. MARCO CASTLLO and J.A. LÓPEZ ORTÍ \\ Departamento de Matemáticas. Universitat Jaume I, Castellón. SPAIN.
}

\begin{abstract}
In order to obtain corrections to orbital elements of minor planets and to determine parameters of rotation of a reference frame, a geometrical model with restrictions in the declination, longitude of the ascending node and argument of the perihelion to minimize a residual function involving all named parameters was presented (Marco et al., 1996, 1997). On the other hand, such rotational model of correction should not modify the semiaxis, eccentricity nor the mean anomaly, so new restrictions on these parameters seem to be necessary. These conditions should reflect the invariability in the size of the orbit and they should be included in the model to complete a consistent set of restrictions.
\end{abstract}

\section{Introduction}

Let $\vec{X}(\alpha, \delta)$ be an unitary vector position in equatorial coordinates for a body in elliptic motion around the Sun. We suppose the existence of errors on its position which are modeled by means of a rotational model given by

$$
\Delta \vec{X}=R \vec{X} \text { with } R=\left[\begin{array}{ccc}
1 & -\Delta \xi & -\Delta \eta \\
\Delta \xi & 1 & -\Delta \varepsilon \\
\Delta \eta & \Delta \varepsilon & 1
\end{array}\right]
$$

From (1) the incremental errors induced by the rotational model and relating the spherical coordinates at whatever time $t$ are given by

$$
\begin{aligned}
\Delta \alpha & =\Delta \xi+\Delta \eta \sin \alpha \tan \delta-\Delta \varepsilon \cos \alpha \tan \delta \\
\Delta \delta & =\Delta \eta \cos \alpha+\Delta \varepsilon \sin \alpha
\end{aligned}
$$

\section{Rotation Model Applied to the Orbital Plane at $t_{0}$}

We denote the different unitary vectors ( at $t_{0}$ ) defining the orbit orientation by $\overrightarrow{p_{1}^{0}}$ (in the perihelion direction), $\overrightarrow{p_{3}^{0}}$ (normal to the orbit) and $\overrightarrow{p_{2}^{0}}$ the necessary one to built a direct triad. The application of the rotational model to the triad is carried out by means of the expressions

$$
R_{1}(\varepsilon) R R_{1}(-\varepsilon) \overrightarrow{p_{i}^{0}}=\overrightarrow{p_{i}^{0}}+\overrightarrow{\Delta p_{i}^{0}} \quad \text { for } \mathrm{i}=1,2,3
$$


where $R$ is the rotation matrix from (1) and $R_{1}( \pm \varepsilon)$ the rotation matrix relating equatorial and ecliptic coordinates. The relation (4) follows from (3)

$$
\overrightarrow{\Delta p_{i}^{0}}=\overrightarrow{p_{i}^{0}} \times R_{1}(\varepsilon) \vec{Y} \text { being } \vec{Y}=[\Delta \varepsilon,-\Delta \eta, \Delta \xi]^{t}
$$

If we express these $\overrightarrow{p_{i}^{0}}$ vectors in ecliptic heliocentric coordinates, due to the fact that they are functions of the incremental values $\Delta \Omega_{0}$ (argument of ascending node), $\Delta \omega_{0}$ (argument of perihelion) and $\Delta i_{0}$ (inclination of the orbit), we can obtain from (3)

$\left[\begin{array}{c}\Delta \varepsilon \\ -\Delta \eta \\ \Delta \xi\end{array}\right]=\left[\begin{array}{ccc}0 & \sin i_{0} \sin \Omega_{0} & \cos \Omega_{0} \\ -\sin \varepsilon & \cos i_{0} \sin \varepsilon+\sin i_{0} \cos \Omega_{0} \cos \varepsilon & -\sin \Omega_{0} \cos \varepsilon \\ \cos \varepsilon & \cos i_{0} \cos \varepsilon-\sin i_{0} \cos \Omega_{0} \sin \varepsilon & \sin \Omega_{0} \sin \varepsilon\end{array}\right]\left[\begin{array}{c}\Delta \Omega_{0} \\ \Delta \omega_{0} \\ \Delta i_{0}\end{array}\right]$

\section{Necessary Compatibility Conditions for the Rotational Model}

Let $\vec{W}=P \vec{v}$ be the ecliptic coordinates of the unitary vector position $\vec{v}=$ $[\cos u, \sin u, 0]^{t}$ in the orbital plane at $t$, where $u$ represents the true anomaly and let $\vec{X}=R_{1}(-\varepsilon) \vec{W}$ be its equatorial representation. Then, we have the incremental vector $\overrightarrow{\Delta W}=R_{1}(\varepsilon) \overrightarrow{\Delta X}$,

$\overrightarrow{\Delta W}=\left[\overrightarrow{\Delta p_{i}} v_{i}+\overrightarrow{p_{i}} \Delta v_{i}\right]$

Also, from (4) this infinitesimal vector is expressed as follows

$$
\overrightarrow{\Delta W}=R_{1}(\varepsilon)[\vec{X} \times \vec{Y}]=\vec{W} \times R_{1}(\varepsilon) \vec{Y}=P \vec{v} \times R_{1}(\varepsilon) \vec{Y}
$$

Through the expression of $P \vec{v}$ in vectorial form and due to the fact that $R_{1}(\varepsilon) \vec{Y}$ is an infinitesimal vector we obtain

$$
\overrightarrow{\Delta W}=\sum_{i=1}^{2} \overrightarrow{p_{i}} v_{i} \times R_{1}(\varepsilon) \vec{Y} \simeq \sum_{i=1}^{2} \overrightarrow{p_{i}^{0}} v_{i} \times R_{1}(\varepsilon) \vec{Y}
$$

applying (5) and (6), we obtain

$$
\overrightarrow{\Delta W}=\sum_{i=1}^{2}\left\{\left[\frac{\overrightarrow{\partial p_{i}}}{\partial \Omega} \Delta \Omega+\frac{\overrightarrow{\partial p_{i}}}{\partial \omega} \Delta \omega+\frac{\overrightarrow{\partial p_{i}}}{\partial i} \Delta i\right] v_{i}+\overrightarrow{p_{i}} \Delta v_{i}\right\}
$$

which give us together with (8) the complete set of restrictions.

\section{References}

F.J. Marco, J.A. López, M-J. Martínez: 1996, A time-dependent extension to Brower's method of orbital elements correction, Dynamics, Ephemerides and Astrometry of the Solar System, pp.199. 202

F.J. Marco, J.A. López, M.J. Martínez: 1997, Temporal variation of perturbed elliptic elements: a semi-analytical approach, Celest. Mech., 68, 193-198 